\title{
ATLAS Higgs and Supersymmetry Physics Prospects at the High-Luminosity LHC
}

\author{
N. Venturi*† \\ CERN \\ E-mail: nicola.venturiecern.ch
}

In the second half of 2026, after a shutdown period of two and one half years for a major upgrade of the accelerators infrastructure, the Large Hadron Collider (LHC) will enter the high-luminosity phase reaching an instantaneous luminosity of $7.5 \times 10^{34} \mathrm{~cm}^{-2} \mathrm{~s}^{-1}$ at the centre-of-mass energy of $14 \mathrm{TeV}$. To cope with those unprecedented luminosities and to be able to fully exploit the physics discovery potential, the ATLAS detector will be upgraded as well. A total of $3000 \mathrm{fb}^{-1}$ of $p p$ collision data should be collected over about ten years of high luminosity LHC running. In this document, simulated data studies on the ultimate precision attainable on the couplings measurements of the $125 \mathrm{GeV}$ Higgs boson, the prospect on the sensitivities for Higgs boson rare decays as well as perspectives on the measurement of the Standard Model di-Higgs production, are presented. Moreover, an overview of the expected sensitivities of the upgraded ATLAS detector to the discovery of several supersymmetric particles production are discussed.

EPS-HEP 2017, European Physical Society conference on High Energy Physics

5-12 July 2017

Venice, Italy

* Speaker.

${ }^{\dagger}$ On behalf of the ATLAS Collaboration 


\section{Introduction}

The High-Luminosity LHC (HL-LHC)[1] phase is scheduled to start mid 2026 and after 10 years of run about $3000 \mathrm{fb}^{-1}$ of $p p$ collision data will be collected at the centre-of-mass energy of $14 \mathrm{TeV}$. The peak instantaneous luminosity will reach an unprecedented value of $7.5 \times$ $10^{34} \mathrm{~cm}^{-2} \mathrm{~s}^{-1}$ which implies an average number of inelastic collisions per bunch crossing (pile-up) of $\left\langle\mu_{P U}\right\rangle \approx 200$. To be able to cope with that unprecedented instantaneous luminosity and pileup conditions and to replace radiation damaged subdetectors, the ATLAS detector [2] will undergo a major upgrade [3]. In particular, the inner tracking detector will be replaced with a new allsilicon trackers with higher granularity and extended coverage in pseudorapidity, $\eta$, up to $|\eta| \approx 4$, the innermost muon barrel detector will be upgraded for increasing triggering capabilities and the readout electronics of the trigger and data acquisition system will be replaced to achieve recording data rates up to $10 \mathrm{kHz}$. In order to maximise the physics performance and discovery potential of ATLAS and to probe different detector upgrade layouts, several benchmark simulated analyses have been performed. The physics analyses are mainly carried out using generator-level MonteCarlo (MC) samples for which the detector response is simulated with smearing and efficiency functions. Those are derived from limited fully-simulated samples using different possible ATLAS HL-LHC detector layouts and high pile-up conditions and are applied as a function of the $p_{T}$ and $\eta$ of the reconstructed physics object. A second approach to avoid too computing-intense MC samples generation, is the extrapolation of the Run-1 and Run-2 results assuming similar detector performances and analysis approaches as the current ones but scaling the signal and background level to the higher luminosity and the increased centre-of-mass energy.

\section{Higgs boson physics prospects at HL-LHC}

The data collected at the end of the HL-LHC will allow for more accurate and detailed studies of the Higgs boson reaching the sensitivity for di-Higgs processes. All the Higgs boson prospect results presented in this section assume a Standard Model (SM) Higgs boson with a mass of $125 \mathrm{GeV}$.

\subsection{Higgs boson couplings}

The measurements of the cross sections times branching ratios are expressed as signal strengths $\mu=\sigma / \sigma_{S M}$ reflecting deviation from the SM predictions. The prospect on the signal strength precision measurements [5] are given in terms of relative uncertainties on $\mu$ and are summarised in figure 1 for $300 \mathrm{fb}^{-1}$, the amount of data expected before the Phase-II ATLAS upgrade, and 3000 $\mathrm{fb}^{-1}$ with a pile up of $\left\langle\mu_{P U}>\approx 140\right.$. The improvements at high luminosity are as large as a factor of 2-3. Those results are then interpreted in terms of Higgs boson couplings to the elementary particles using a leading order motivated framework [4] within a narrow width approximation. Assuming a model with no new Higgs boson decay modes, the mass dependence of the reduced coupling constants of several SM particles and their expected precisions are shown in figure 1 and range from $3-4 \%$ for the $\mathrm{W}, \mathrm{Z}$ bosons to $8-12 \%$ for the top, b quark and tau lepton.

\subsection{Higgs boson self-coupling}

Of particular interest at the HL-LHC is the measurement of the Higgs boson pair production from which one can test the trilinear Higgs boson self coupling, $\lambda_{H H H}$ and hence probe 

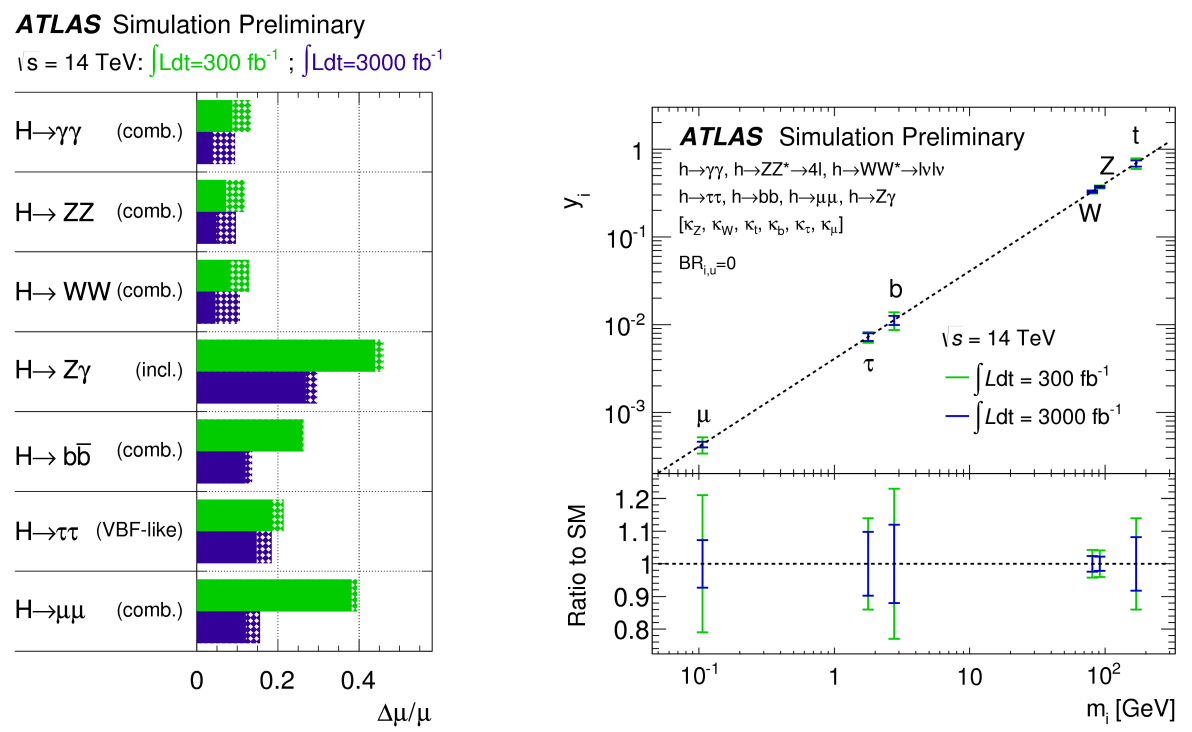

Figure 1: Right: Expected relative uncertainty on the signal strength $\mu$ for several Higgs boson final states expected with 300 and $3000 \mathrm{fb}^{-1}$ of $p p$ data. The hashed areas indicate the increase of the estimated error due to current theory systematic uncertainties [5]. Left: expected fit results for the reduced coupling scale factors (y-axis) for SM fermions and bosons masses (x-axis) compared to the predicted mass dependence for the SM Higgs boson (dashed line) [5].

the form of the Higgs potential. There are two processes interfering destructively generating the di-Higgs boson final state, one of them depending on the Higgs boson triple-linear self coupling giving an expected NNLO total production cross section of $41 \mathrm{fb}^{-1}$ at $14 \mathrm{TeV}$ [6]. Due to their high branching ratios the decay channels involving b-quarks are of great importance. With the upgraded inner detector and the extension in pseudorapidity coverage, it will be possible to achieve a b-tag efficiency compatible with the actual one even under extreme pile-up condition as those of the HL-LHC [7]. Among the most promising signature there is the $H H \rightarrow b \bar{b} \gamma \gamma$ with 9.5 expected events in the signal region giving a $1.05 \sigma$ statistical significance. This channel will set a constraint in the deviation of the Higgs self coupling from the SM in the range of $-0.8<\lambda_{H H H} / \lambda_{S M}<7.7$ at $95 \%$ C.L. neglecting all systematics [8]. From the $H H \rightarrow b \bar{b} b \bar{b}$ decay channel the constraint will be $0.2<\lambda_{H H H} / \lambda_{S M}<7.0$ at $95 \%$ C.L. (without systematic uncertainties) and $-3.5<\lambda_{H H H} / \lambda_{S M}<11.0$ if current systematics are assumed [9]. Further decay channels that are exploited are $H H \rightarrow b b \tau \tau$ [10] with an expected significance of $0.60 \sigma$ and constraining the self coupling to $-4.0<\lambda_{H H H} / \lambda_{S M}<12.0$ and $t \bar{t} H H \rightarrow W b W b b \bar{b} b \bar{b}$ with a statistical significance of $0.35 \sigma$ [11]. To achieve the maximal sensitivity to the Higgs boson self-coupling all measurable channels will be statistically combined.

\subsection{Vector Boson Fusion Higgs boson production and Higgs boson rare decays}

The large data sample available after the HL-LHC phase will allow to probe the couplings to the second generation of fermions. The expected statistical significance of the $H \rightarrow \mu \mu$ decay channel will improve from $2.3 \sigma$ with $300 \mathrm{fb}^{-1}$ to $7.0 \sigma$ with $3000 \mathrm{fb}^{-1}$ assuming a pile-up of $<\mu_{P U}>\approx 140$ with a relative precision on the signal strength of $\delta \mu / \mu=0.2$ [7]. This channel will 
also benefit from the improved mass resolution of around $25 \%$ offered by the fully-silicon inner detector upgrade contributing to the precise determination of the Higgs boson mass. Projections for the measurement of $H \rightarrow J / \Psi \gamma$ decay have also been performed [12]. The expected yields are 3 signal and 1700 background events setting the upper limit on the branching ratio to $44_{-22}^{+19} 10^{-6}$ at $95 \%$ C.L. (SM prediction is $2.9 \pm 0.210^{-6}$ ). The extended pseudorapidity coverage of the new inner tracker will also allow an efficient pile-up suppression from about 200 pile-up jets per event down to 0.2 which is particular beneficial for the vector boson fusion (VBF) Higgs boson production topologies characterised by two hard forward jets [7]. This efficient pile-up mitigation will allow to measure the cross-section of the $H \rightarrow W W^{*}$ with an expected precision of $12 \%$ whereas $H \rightarrow Z Z^{*}$ decay mode measurement will have a statistical significance of $7.7 \sigma$ and an expected signal strength precision of $\Delta \mu / \mu=0.17$.

\section{Supersymmetry prospects at HL-LHC}

Supersymmetry (SUSY) $[13,14,15,16,17,18]$ is an appealing possible extension of the SM which predicts the existence of a scalar partner to the SM fermion and a fermionic partner to the SM boson. The discovery or more stringent limits on the weak-scale SUSY particles is one of the priorities for the future HL-LHC physics program as the sensitivities will be strongly enhanced at the LHC design centre-of-mass energy. Discovery and exclusion projections have been studied for several benchmark SUSY scenarios at the HL-LHC with pile up condition of $\left\langle\mu_{P U}>\approx 200\right.$ using a total integrated luminosity of $3000 \mathrm{fb}^{-1}$ of data. The analyses presented assume a systematic uncertainty of $30 \%$ on the background estimation.
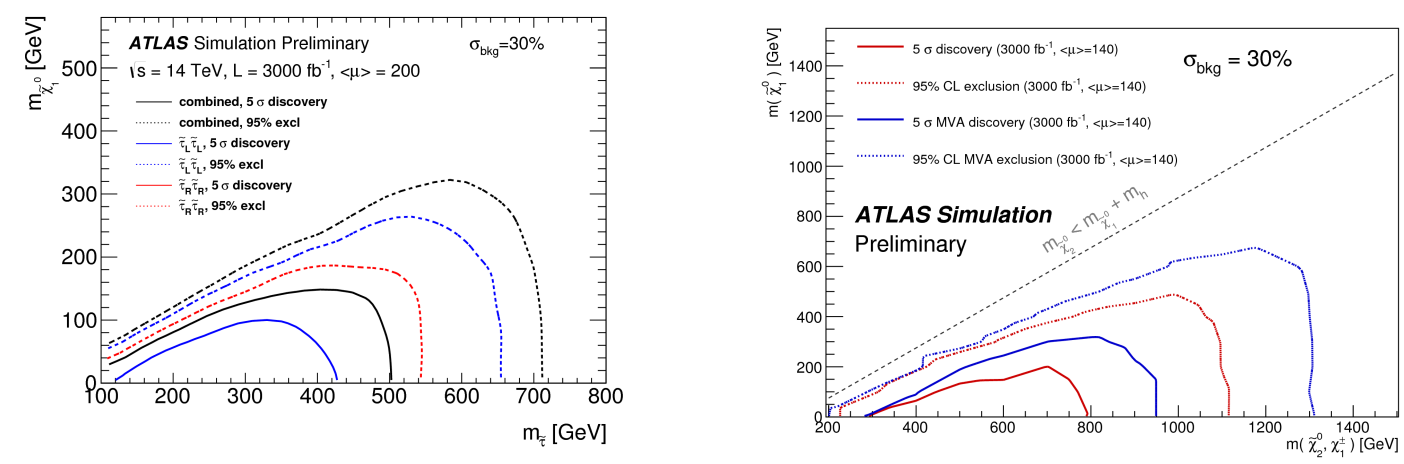

Figure 2: Right: $95 \%$ C.L. exclusion limits and $5 \sigma$ discovery contours on pure $\tilde{\tau}_{L}, \tilde{\tau}_{L}$, pure $\tilde{\tau}_{R}, \tilde{\tau}_{R}$ and combined tau slepton production mode [20]. Left: 95\% C.L. exclusion limits and $5 \sigma$ discovery contours in the lightest neutralino $\tilde{\chi}_{1}^{0}$ versus charginos $\tilde{\chi}_{2}^{ \pm}$and neutralino $\tilde{\chi}_{2}^{0}$ mass plane for the cut and count and the multivariate analysis approaches [21].

\subsection{Scalar top quark pair production}

The on-going searches at the LHC have limited sensitivity to the scalar top quark production for the case in which its mass difference with the neutralino is the SM top quark mass. The semileptonic final state is very similar to the SM $t \bar{t}$ with enhanced missing transverse energy coming from the stable neutralinos. The discovery projection at the HL-LHC will be up to $450 \mathrm{GeV}$ and the exclusion limit will be increased up to $700 \mathrm{GeV}$ for the stop mass eigenstate [19]. 


\subsection{Scalar tau lepton pair production}

Electro-weak SUSY production of scalar tau lepton is an important channel for testing the reconstruction and identification of tau's with the upgraded ATLAS detector. Also, in many SUSY scenarios, light scalar taus can affect the decay rate of the Higgs boson into di-photons. The final state considered here involves two hadronically decaying taus, low jet activity and large missing transverse energy. The $5 \sigma$ discovery potential and $95 \%$ C.L. exclusion is shown in figure 2 for the pure left and right handed mass scalar tau production and for their combinations [20]. For the latter case, the exclusion range reaches $700 \mathrm{GeV}$ and the discovery ranges from 100 up to $500 \mathrm{GeV}$ in scalar tau mass.

\subsection{Direct chargino and neutralino pair production}

Several SUSY models predict charginos and neutralino to have masses of hundreds of $\mathrm{GeV}$ so within the discovery reach of the LHC. One of those model, simplified with several assumptions on the masses of scalar leptons and scalar neutrinos and assuming equal masses for the charginos $\tilde{\chi}_{2}^{ \pm}$, and neutralino $\tilde{\chi}_{2}^{0}$, has been studies. The very challenging final state consists of leptons, missing transverse energy and $b$-jets allowing also to test the future detector performances under pile-up condition of $\left\langle\mu_{P U}>\approx 140\right.$. The expected $95 \%$ C.L. exclusion and the $5 \sigma$ discovery contours for the cut based analysis and the multivariate analysis can be seen in figure 2. For a massless lightest neutralino $\tilde{\chi}_{1}^{0}$ the discovery contour is extended up to $950 \mathrm{GeV}$ and the exclusion limit up to 1350 $\mathrm{GeV}$ in charginos and neutralino masses [21].

\section{Conclusions}

The HL-LHC phase will deliver $3000 \mathrm{fb}^{-1}$ of data at the centre-of-mass energy of $14 \mathrm{TeV}$ reaching an unprecedented instantaneous luminosity of $7.5 \times 10^{34} \mathrm{~cm}^{-2} \mathrm{~s}^{-1}$ with about 200 pile-up interactions per bunch-crossing. The ATLAS detector will undergo a major upgrade to maintain the current optimal performances hence allowing the full exploitation of the physics potential of the HL-LHC data. Several prospect physics analyses and performance studies have been performed for the Phase-II detector upgrade assuming different detector geometries and designs and pileup conditions. In particular, the precision measurements of the SM Higgs boson couplings to SM particles (from $2 \%$ to 15\%), the expected sensitivity of the Higgs boson self-coupling measurement and to rare Higgs boson decay channels have been explored. Moreover, the discovery potential of several SUSY particles such as scalar top quark, scalar tau lepton, charginos and neutralinos has been explored. All the analyses performed so far in the context of the physics prospects at the HL-LHC will greatly benefit from the Phase-II ATLAS upgrade and the large dataset collected.

\section{References}

[1] G. Apollinari, I. Béjar Alonso, O. Brüning, M. Lamont, L. Rossi, High-Luminosity Large Hadron Collider (HL-LHC): Preliminary Design Report, CERN-2015-005.

[2] ATLAS Collaboration, The ATLAS Experiment at the Large Hadron Collider, JINST 3 (2008) S08003.

[3] ATLAS Collaboration, Letter of Intent for the Phase-II Upgrade of the ATLAS Experiment, CERN-LHCC-2012-022. 
[4] LHC Higgs Cross Section Working Group, Handbook of of LHC Higgs Cross Sections: 3. Higgs Properties, [hep-ph/1307.1347].

[5] The ATLAS Collaboration, Projections for measurements of Higgs boson signal strengths and coupling parameters with the ATLAS detector at the HL-LHC, ATL-PHYS-PUB-2014-016.

[6] D. de Florian and J. Mazzitelli, Higgs Boson Pair Production at Next-to-Next-to-Leading Order in QCD, Phys. Rev. Letter 111 (2013) 201801.

[7] ATLAS Collaboration, Technical Design Report for the ATLAS Inner Tracker Strip Detector, ATL-TDR-025 LHCC-017-055.

[8] ATLAS Collaboration, Study of the double Higgs production channel $H(\rightarrow b \bar{b}) H(\rightarrow \gamma \gamma)$ with the ATLAS experiment at the HL-LHC, ATL-PHYS-PUB-2017-001.

[9] ATLAS Collaboration, Projected sensitivity to non-resonant Higgs boson pair production in the bbbb final state using proton-proton collisions at HL-LHC with the ATLAS detector, ATL-PHYS-PUB-2016-024.

[10] ATLAS Collaboration, Higgs Pair Production in the $H(\rightarrow \tau \tau) H(\rightarrow b \bar{b})$ channel at the High-Luminosity LHC, ATL-PHYS-PUB-2015-046.

[11] ATLAS Collaboration, Prospects for Observing ttHH Production with the ATLAS Experiment at the HL-LHC, ATL-PHYS-PUB-2016-023.

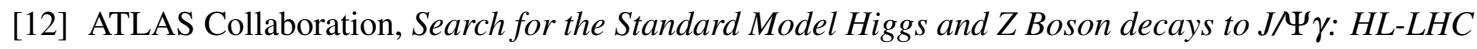
projections, ATL-PHYS-PUB-2015-043.

[13] Yu. A. Golfand and E. P. Likhtman,Extension of the Algebra of Poincare Group Generators and Violation of P Invariance, JETP Lett. 13 (1971) 323, [Pisma Zh. Eksp. Teor. Fiz.13,452(1971)].

[14] D. V. Volkov and V. P. Akulov, Is the Neutrino a Goldstone Particle?, Phys. Lett. B46 (1973) 109.

[15] J. Wess and B. Zumino, Supergauge Transformations in Four-Dimensions, Nucl. Phys. B70 (1974) 39.

[16] J. Wess and B. Zumino, Supergauge Invariant Extension of Quantum Electrodynamics, Nucl. Phys. B78 (1974) 1.

[17] S. Ferrara and B. Zumino, Supergauge Invariant Yang-Mills Theories, Nucl. Phys. B79 (1974) 413.

[18] A. Salam and J. A. Strathdee, Supersymmetry and Nonabelian Gauges, Phys. Lett. B51 (1974) 353.

[19] ATLAS Collaboration, Prospects for a search for direct pair production of top squarks in scenarios with compressed mass spectra at the high luminosity LHC with the ATLAS Detector, ATL-PHYS-PUB-2016-022.

[20] ATLAS Collaboration, Prospect for a search for direct stau production in events with at least two hadronic taus and missing transverse momentum at the high luminosity LHC with the ATLAS Detector, ATL-PHYS-PUB-2016-021.

[21] ATLAS Collaboration, Prospect for a search for direct pair production of a chargino and a neutralino decaying via $a \mathrm{~W}$ boson and the lightest Higgs boson in final states with one lepton, two b-jets and missing transverse momentum at the high luminosity LHC with the ATLAS Detector, ATL-PHYS-PUB-2015-032. 